BONPLANDIA 17(1): 13-24. 2008

\title{
NEW SPECIES OF BACCHARIS (ASTERACEAE, ASTEREAE) FROM BRAZIL
}

\author{
ANABELA SILVEIRA DE OLIVEIRA-DEBLE${ }^{1} \&$ LEONARDO PAZ DEBLE²
}

\begin{abstract}
Summary: Oliveira-Deble, A. S. de \& Deble, L. P. 2008. New species of Baccharis (Asteraceae, Astereae) from Brazil. Bonplandia 17(1): 13-24. ISSN: 0524-0476.

In a recent review of the genus Baccharis $L$. we recognized five new species: Baccharis crassipappa Deble \& Oliveira-Deble, B. inexspectata Deble \& Oliveira-Deble, $B$. multipaniculata Oliveira-Deble \& Deble, $B$. nassauvioides Oliveira-Deble \& Deble and $B$. suberectifolia Oliveira-Deble \& Deble. All species are described and illustrated and delimitation with allied taxa is discussed.
\end{abstract}

Key words: Baccharis, taxonomy, new species, Asteraceae, Brazil.

Resumen: Oliveira-Deble, A. S. de \& Deble, L. P. 2008. Nuevas especies de Baccharis (Asteraceae, Astereae) para Brasil. Bonplandia 17(1): 13-24. ISSN: 0524-0476.

En una revisión reciente del género Baccharis L. para Brasil, fueron reconocidas cinco especies nuevas: Baccharis crassipappa Deble \& Oliveira-Deble, B. inexspectata Deble \& Oliveira-Deble, B. multipaniculata Oliveira-Deble \& Deble, B. nassauvioides Oliveira-Deble \& Deble y $B$. suberectifolia Oliveira-Deble \& Deble. Todas las especies son descriptas, ilustradas y diferenciadas de los taxones relacionados.

Palabras clave: Baccharis, taxonomía, nuevas especies, Asteraceae, Brasil.

Introduction

The New World genus Baccharis L. comprises between 400 and 500 species, frequently dioecius or imperfectly dioecius, of perennial herbs, vines, subshrubs, shrubs, or small trees (Oliveira-Deble, 2008). In a recent check list for Brazil 146 species were accepted (Oliveira \& al., 2006). Among the main diversity centers within the country there are the grasslands and open forests of the southern Brazil as well as the highest elevations of the Brazilian Plateau, especially in the southeast and in part of northeast regions (Bahia). On the other hand, the lesser species frequency is observed in the middle west, northeast and north of Brazil. During a the review of the genus for Brazil we have recognized five new species that are here described and illustrated, and the delimitation with its nearest relatives is discussed.

\footnotetext{
${ }^{1}$ Departamento de Ciências Florestais, Universidade Federal de Santa Maria, CEP: 97.050-110, Santa Maria, RS, Brazil. E-mail: anabela.biol@gmail.com

${ }^{2}$ Departamento de Ciências Florestais, Universidade Federal de Santa Maria, CEP: 97.050-110, Santa Maria, RS, Brazil. E-mail: deble.biol@gmail.com
} 


\section{Results}

\section{Baccharis crassipappa Deble \& Oliveira- Deble, sp. nov.}

Fig. 1

Suffrutex 0,5 m altus; caulibus ramosus, adscendentibus erectisve. Folia linearia, anguste oblonga vel anguste oblanceolata, $15-50 \mathrm{~mm}$ longa, 1-2 mm lata, alterna (internodiis 1-5 mm longis), uninervata, sessilia, concolora, integra, glabrata vel pauci pilosa, apice leviter acuta ad rotundata, basi attenuata. Capitula (breviter) pedicellata, racemosa vel solitaria in axillis foliorum disposita. Capitula feminea campanulata, 4-4,5 mm alta et crassa; involucrum 3-4 mm altum, 4-4,5 mm crassum, 18-24-florum. Bracteis involucri 4-seriatis, leviter acutis, dorso brunneis, margine hyalinis; externis ovatis ad ovato-lanceolatis, 1,2-2 $\mathrm{mm}$ longis, 0,8-1,2 $\mathrm{mm}$ latis; mediis lanceolatis, 2,5-3 mm longis, 0,8-1 $\mathrm{mm}$ latis; internis lanceolatis, 2,8-3,3 mm longis, 0,7-0,9 mm latis. Flores feminei corolla tubulosa, 1,7-1,9 mm longa, apice 5-dentata. Stylo 2-2,2 mm longo; rami styli lanceolati, 0,4 mm longi. Pappus albus, multiseriatus, 2-2,2 mm longus; setis pappi 60-70. Achaenia sordescens, 10-costata, glabrata, obovata, 1-1,2 mm longa. Capitula mascula campanulata, 2,5-3,5 $\mathrm{mm}$ alta, 4-4,5 $\mathrm{mm}$ crassa; involucrum 2,5-3 $\mathrm{mm}$ altum, 3,5-4 $\mathrm{mm}$ crassum, 17-19-florum. Bracteis involucri 4-seriatis, leviter acutis, dorso brunneis, margine hyalinis; externis ovatis vel ovato-lanceolatis, $1,5-2 \mathrm{~mm}$ longis, $0,8-$ $1 \mathrm{~mm}$ latis; mediis lanceolatis, 2-2,5 mm longis, 0,8-1 mm latis; internis anguste lanceolatis, 2-2,8 $\mathrm{mm}$ longis, 0,6-0,7 $\mathrm{mm}$ latis. Flores mascula corolla tubuloso campanulata, 2-2,5 mm longa, apice 5-lobata; lobulis lanceolatis, 0,6-0,8 mm longis. Antherae 0,6-0,8 mm longae. Stylus 2-2,3 $\mathrm{mm}$ longus; rami styli breves, connati. Pappus albus, uniseriatus, 1,8-2,2 mm longus; setis pappi 21-27.

Typus: Brazil. Minas Gerais: Joaquim Felício, Serra do Cabral, subarbusto de $50 \mathrm{~cm}$, capítulo alvascento, margem do córrego encachoeirado, campo serrado, exemplar + , 10-IV-2004, G. Hatschbach, M. Hatschbach \& O. S. Ribas 77696 (holotypus MBM).
Shrub 0.5 tall; branching erect-patent. Leaves linear, narrowly oblong to narrowly oblanceolate, 15-50 mm long, 1-2 mm wide, alternate, internodes 1-5 mm long, appearing uninervate, sessile, concolorous, margin entire, apex slightly acute to rounded, base narrowed; both leaf surfaces (and shoots) with tufted indument of flagellate hairs, usually covered by resin. Capitula (shortly) pedunculate, arranged in terminal racemes or solitary in the leaf axils; peduncles up to 25 mm long; bracts 0-2. Female capitula campanulate, 4-4.5 mm long and wide; flowers 18-24; involucre 3-4 mm long, 4-4.5 mm wide; involucral bracts in 4 series, apex slightly acute, darker in the distal third, margin hyaline; outer bracts ovate to ovatelanceolate 1.2-2 mm long, 0.8-1.2 mm wide; median bracts lanceolate, $2.5-3 \mathrm{~mm}$ long, 0.8$1 \mathrm{~mm}$ wide; inner bracts lanceolate, 2.8-3.3 $\mathrm{mm}$ long, $0.7-0.9 \mathrm{~mm}$ wide. Female corolla tubular, 1.7-1.9 mm long, apically with 5 short teeth. Style slightly exceeding the corolla, 2-2.2 mm long; branches lanceolate, $0.4 \mathrm{~mm}$ long. Pappus white, in several series, 2-2.2 mm long; bristles 60-70. Achenes obovoid, 1-1.2 mm long, glabrous, brownish, 10-ribbed. Male capitula campanulate, 2.5$3.5 \mathrm{~mm}$ long, 4-4.5 mm wide; flowers 17-19; involucre 2.5-3 mm long, 3.5-4 mm wide; involucral bracts in 4 series, apex slightly acute, darker in the distal third, margin hyaline; outer bracts, ovate to ovatelanceolate, 1.5-2 mm long, 0.8-1 mm wide; median bracts, lanceolate, $2-2.5 \mathrm{~mm}$ long, 0.8-1 mm wide; inner bracts, narrowly lanceolate, 2-2.8 mm long, 0.6-0.7 mm wide. Male corolla 2-2.5 mm long, apically with 5 lanceolate lobes, 0.6-0.8 mm long. Anthers $0.6-0.8 \mathrm{~mm}$ long. Style not or slightly exceeding the corolla, 2-2.3 mm long; style branches short, attached. Pappus white, uniseriate, 1.8-2.2 mm long; bristles 21-27.

Distribution and habitat: Baccharis crassipappa is known only from the type population located at the Serra do Cabral (Joaquim Felício), in north central of Minas Gerais (Brazil).

Obs.: Baccharis crassipappa is similar to 


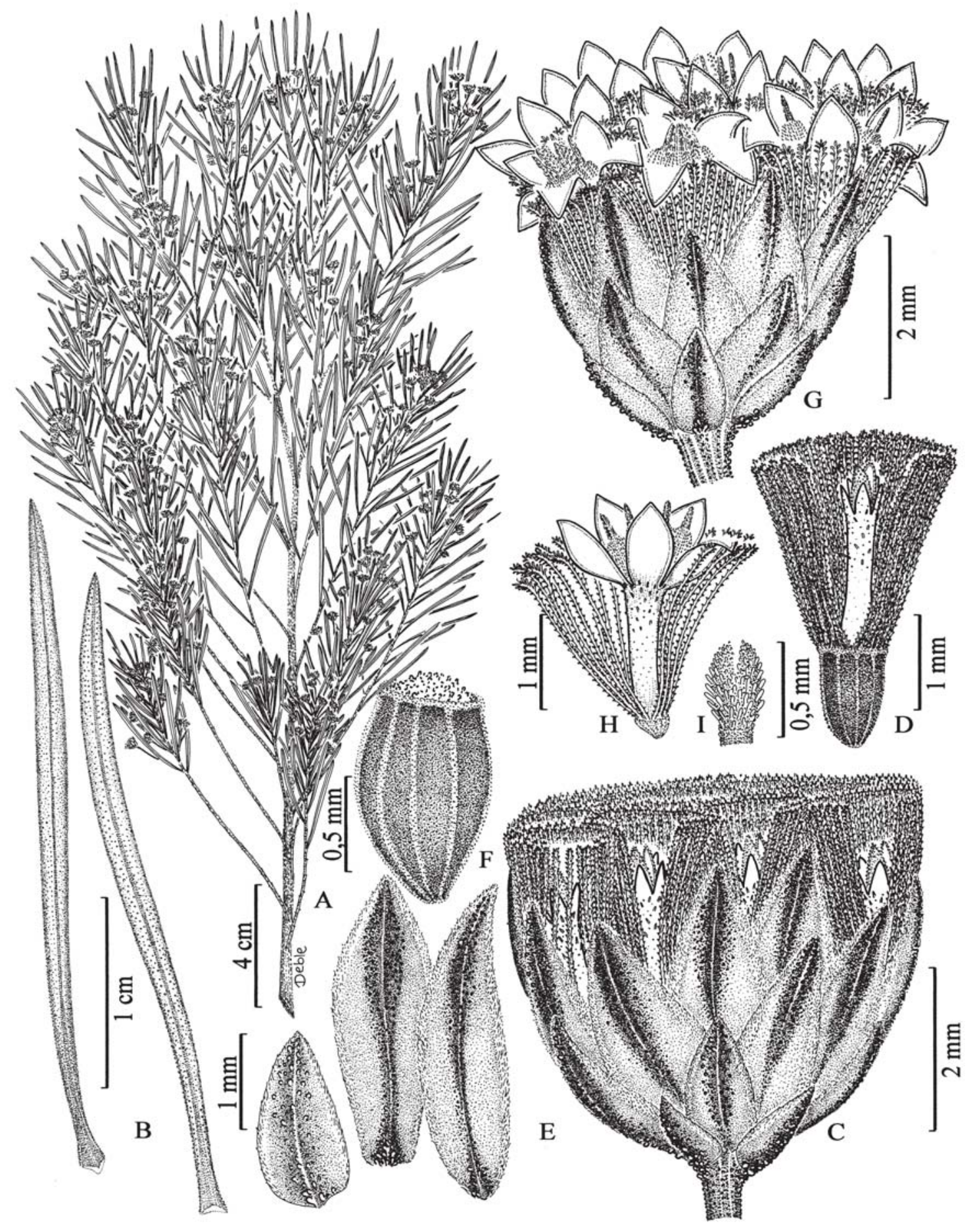

Fig. 1. Baccharis crassipappa Deble \& Oliveira-Deble. A: male branch. B: leaves. C: female capitulum. D: female flower. E: female involucral bracts. F: achene. G: male capitulum. H: male flower. I: apex of style from male flower (A, B, G-I, Hatschbach \& al. 77.699 paratypus MBM; C-F, Hatschbach \& al. 77696 holotypus MBM). 
Baccharis myriciifolia DC., but differs by entire, 1-2 mm wide leaves (versus leaves frequently with 2-5 obtuse teeth, and 3-10 mm wide), as well as by thicker pappus bristles of female flowers.

Additional specimen examined. BRAZIL. Minas Gerais: Joaquim Felício, Serra do Cabral, subarbusto de $50 \mathrm{~cm}$, capítulo alvascento, margem do córrego encachoeirado, campo serrado, exemplar $\sigma^{x}, 10-I V-2004$, G. Hatschbach, M. Hatschbach \& O. S. Ribas 77699 (MBM).

Etymology: The specific epithet refers to the thick pappus bristles.

\section{Baccharis inexspectata Deble \& Oliveira-Deble, sp. nov.}

Fig. 2

Frutex ramosus, 0,5-2 m altus; caulibus adscendentibus erectisve, usque ad apice foliosus. Folia anguste oblonga vel obovata, 3-15 mm longa, 0,8-6 mm lata, alterna (internodiis 1-5 mm longis), 1-3-nervata, sessilia, concolora, integra vel utrinque 1-3-dentata, punctulato-glandulosa, apice obtusa vel rotunda, basi cuneata vel attenuata. Capitula sessilia vel breviter pedicelata in spicis foliorum disposita. Involucrum femineum cilyndraceum, 4-5 $\mathrm{mm}$ altum, 0,8-1,5 $\mathrm{mm}$ crassum, 2-4-florum. Bracteis involucri 4-5-seriatis, leviter acutis, margine hyalinis; externis ovatis ad ovato lanceolatis, 0,8-2 mm longis, 0,5-0,8 mm latis; mediis ovato lanceolatis ad lanceolatis, 2,5-4 mm longis, 0,7-1 mm latis; intimis oblongis, 3,8-4,3 mm longis, 0,6$1 \mathrm{~mm}$ latis. Flores feminei corolla tubularfiliformia, 1,6-2,2 mm longa, apice dentata. Stylus 2-3,2 mm longus; rami styli lanceolati, 0,4-0,5 $\mathrm{mm}$, longi exserti. Pappus albus, uniseriatus, 2,2$3 \mathrm{~mm}$ longus; setis pappi 45-50. Achaenia glabra, castanea, 10-12-costata, obovato-elliptica, 1-1,2 $\mathrm{mm}$ longa. Involucrum mascularum anguste campanulatum, 3-4,5 $\mathrm{mm}$ altum, 1,2-2 $\mathrm{mm}$ crassum, 5-8-florum. Bracteis involucri 3-4seriatis, acutis; externis ovatis, 1-2 mm longis, 0,6-0,8 mm latis; mediis ovato-lanceolatis, 2,5-3 mm longis, 1-1,2 mm latis; intimis ellipticis, 3,5-4 $\mathrm{mm}$ longis, 0,8-1 mm latis. Flores mascula corolla tubuloso campanulata, 2,8-3 mm longa, apice 5- lobata; lobis lanceolatis, 0,8-1,1 mm longis. Antherae 0,8-1 mm. Stylus 3-3,2 mm longus; rami styli connati. Pappus albus, uniseriatus, 2-2,5 mm longus; setis pappi 18-22.

Typus: Brazil. Rio Grande do Sul: Rosário do Sul, Serra do Caverá, Cerro Grande, 30¹1'49,3”'S-5508'10,4”'W, população próximo ao topo, exemplar ㅇ, 01-V-2007, L. P. Deble \& A. S. de Oliveira-Deble 9344 (holotypus MBM, isotypi CTES, SI).

Shrub 0.5-2 m tall; branching erect-patent or erect, glutinous. Leaves narrowly oblong to obovate, 3-15 mm long, 0.8-6 mm wide, alternate, appearing 1-3-nervate, sessile, glandular, concolorous, margin entire or with 1-3 short or long obtuse teeth, apex obtuse to rounded, base narrowed to cuneate, internodes 1-5 mm long. Capitula sessile or shortly pedunculate, in the leaf axils. Female capitula and involucre narrowly cylindrical, 4-5 mm long, 0.8-1.5 mm wide; flowers 2-4; involucral bracts in 4-5 series, apex slightly acute, margin hyaline; outer bracts, ovate to ovate-lanceolate $0.8-2 \mathrm{~mm}$ long, $0.5-0.8 \mathrm{~mm}$ wide; median bracts, ovate-lanceolate to lanceolate, $2.5-4 \mathrm{~mm}$ long, 0.7-1 mm wide; inner bracts, oblongs, 3.8-4.3 mm long, 0.6-1 $\mathrm{mm}$ wide. Female corolla tubular-filiform, 1.6-2.2 mm long, apically with 5 short teeth. Style exceeding the corolla, 2-3.2 mm long; branches lanceolate, 0.4-0.5 mm long. Pappus white, uniseriate, 2.2-3 mm long; bristles 4550. Achenes obovoid-ellipsoid, 1-1.2 mm long, glabrous, brownish, 10-12-ribbed. Male capitula and involucre narrowly campanulate, 3-4.5 mm long, 1.2-2 mm wide; flowers 5-8; involucral bracts in 3-4 series, apex acute; outer bracts, ovate, 1-2 mm long, 0.6-0.8 mm wide; median bracts, ovate-lanceolate, 2.5-3 mm long, 1-1.2 mm wide; inner bracts, elliptic, 3.5-4 mm long, 0.8-1 mm wide. Male corolla $2.8-3 \mathrm{~mm}$ long, apically with 5 lanceolate lobes, 0.8-1.1 mm long. Anthers 0.8-1 mm long. Style not or slightly exceeding the corolla, 3-3.2 mm long; style branches short, attached. Pappus white, uniseriate, 2$2.5 \mathrm{~mm}$ long; bristles 18-22.

Distribution and habitat: Baccharis 


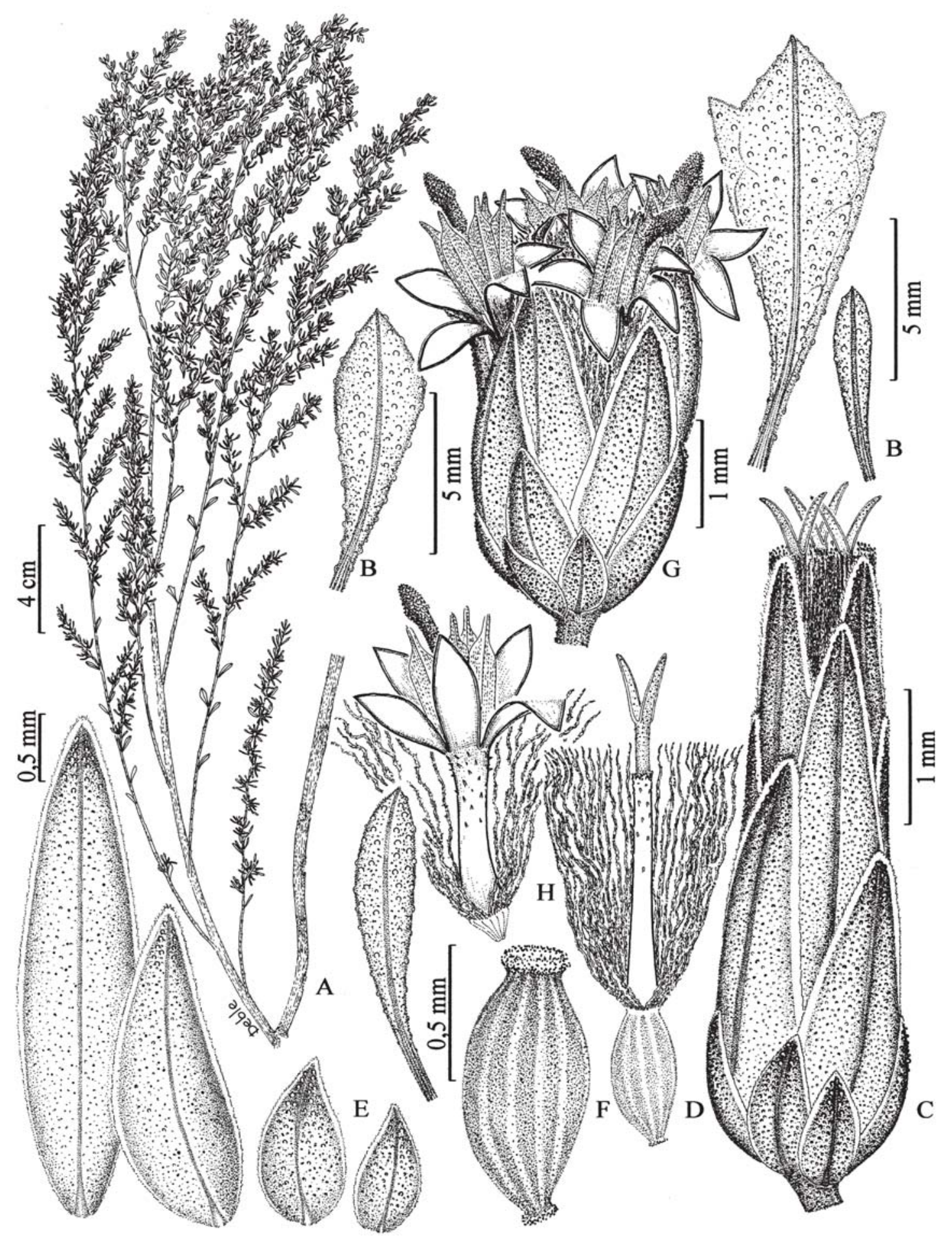

Fig. 2. Baccharis inexspectata Deble \& Oliveira-Deble. A: female branch. B: leaves. C: female capitulum. D: female flower. E: female involucral bracts. F: achene. G: male capitulum. H: male flower (A-F, Deble \& Oliveira-Deble 9344 holotypus MBM; G-H, Deble \& Oliveira-Deble 9345 paratypus MBM). 
inexspectata is known only from the type population at the highest elevations of the Serra do Caverá (Rosário do Sul), in the south of Rio Grande do Sul (Brazil).

Obs.: Baccharis inexspectata belongs to sect. Cylindricae Heering, ser. Axillaris Giuliano. The new species is close to Baccharis perlata Sch.Bip. ex Baker, from Bahia and Minas Gerais, but differs by oblong to obovate leaves, frequently with 1-3 teeth at the margin and 0.8-6 mm wide (versus leaves narrowly spathulate with entire margin and with $0.5-2 \mathrm{~mm}$ wide), by flexible, twisting, and narrowly filiform pappus bristles of female flowers (versus pappus bristles rigid, erect, and filiform), as well as by obovoidellipsoid, 1-1.2 mm long achenes (versus nearly cylindrical with a narrowed base, $2 \mathrm{~mm}$ long achenes).

Additional specimens examined. BRAZIL. Rio Grande do Sul: Rosário do Sul, Serra do Caverá, Cerro Grande, 30¹1’49,3”S-5508'10,4”W, população próximo ao topo, exemplar $0^{7}, 01-\mathrm{V}$ 2007, L. P. Deble \& A. S. de Oliveira-Deble 9345 (MBM, CTES, SI); idem, capítulos masculinos imaturos, 01-V-2007, L. P. Deble \& A. S. de Oliveira-Deble 9346 (MBM).

Etymology: This species is named by the unexpected way in that it was found.

\section{Baccharis multipaniculata Oliveira- Deble \& Deble, sp. nov.}

Fig. 3

Suffrutex ramosus 0,5-1,5 m altus; caulibus pilosus, adscendentibus erectisve. Folia linearlanceolata vel lanceolata 10-25 mm longa, 1-4 $\mathrm{mm}$ lata, subopposita vel alterna, sessilia, uninervata, concolora, integra, revoluta; laminis conspersis pilosis (pilis flageliformis et retrorsis munitis), apice acutis, basi semiamplexicaulis. Capitula pedicellata in multi panicula racemosis disposita. Capitula feminea late campanulata, 2,5-3 $\mathrm{mm}$ alta et crassa; involucrum, 2-2,5 $\mathrm{mm}$ altum, 2,5 mm crassum, 6-7-florum. Bracteis involucri 2-3-seriatis, obtusis vel rotundatis, dor- so brunneis, margine hyalinis; externis ovatis, 1$1,5 \mathrm{~mm}$ longis, 0,7-1 mm latis; mediis et intimis ellipticis, 2-2,5 mm longis, 0,8-1 mm latis. Flores feminei corolla tubulosa, 1,3-1,5 mm longa, apice glanduloso dentata. Stylus 1,5-1,8 mm longus; rami styli lanceolati, 0,2-0,3 $\mathrm{mm}$ longi. Pappus albus, uniseriatus, 1,8-2,2 mm longus; setis pappi 35-40. Achaenia fulva, 5-costata, obovato elliptica, 1-1,2 mm longa, cum pappilis vestita. Capitula mascula hemisphaerica, 2-2,5 $\mathrm{mm}$ alta, 2,5 mm crassa; involucrum 2-2,5 mm altum et crassum, 12-16-florum. Bracteis involucri 2-3seriatis, leviter acutis vel obtusis; externis ovatis, 1-1,6 mm longis, 0,8-1 mm latis; intimis ovatooblongis, 1,5-2 mm longis, 1-1,2 mm latis. Flores mascula corolla tubuloso-campanulata, 1-1,2 mm longa, apice 5-lobata, cum pilis geminis et papillis vestita. Antherae 0,5-0,7 mm longae. Stylus 1-1,5 $\mathrm{mm}$ longus; rami styli breves, connati. Pappus albus, uniseriatus, 1-1,5 mm longus; setis pappi 15-20.

Typus: Brazil. Santa Catarina: Curitibanos, no campo, ㅇ, 900 m s.m., 22-II-1962, R. Reitz \& R. M. Klein 12222 (holotypus HBR).

Subshrub 0.5-1.5 m tall; branching erectpatent, shoots with dense indument of uniseriate hairs and glandular hairs. Leaves linear-lanceolate to lanceolate, $10-25 \mathrm{~mm}$ long, 1-4 mm wide, subopposite to alternate, sessile, appearing uninervate, concolorous; margin entire and revolute, apex acute, base subcordate; both leaf surfaces with uniseriate flagellate hairs, glandular hairs and short rigid pedestal hairs, internodes to $10 \mathrm{~mm}$ long. Capitula pedunculate, borne in dense terminal panicles. Female capitula broadly campanulate, 2.5-3 mm long and wide; flowers 6-7; involucre 2-2.5 $\mathrm{mm}$ long, $2.5 \mathrm{~mm}$ wide. Involucral bracts in 2-3 series, apex obtuse to rounded, often darker in the distal part, margins hyalines; outer bracts ovate 1-1.5 mm long, 0.7-1 mm wide; median and inner bracts broadly elliptic, 2-2.5 mm long, 0.8-1 mm wide. Female corolla tubular, 1.3-1.5 mm long, apically with 5 papillose teeth. Style slightly exceeding the corolla, 1.5-1.8 mm long; branches lanceolate, $0.2-0.3 \mathrm{~mm}$ long. Pappus white, uniseriate, 1.8-2.2 mm long; bristles 35-40, basally connate, persistent. 


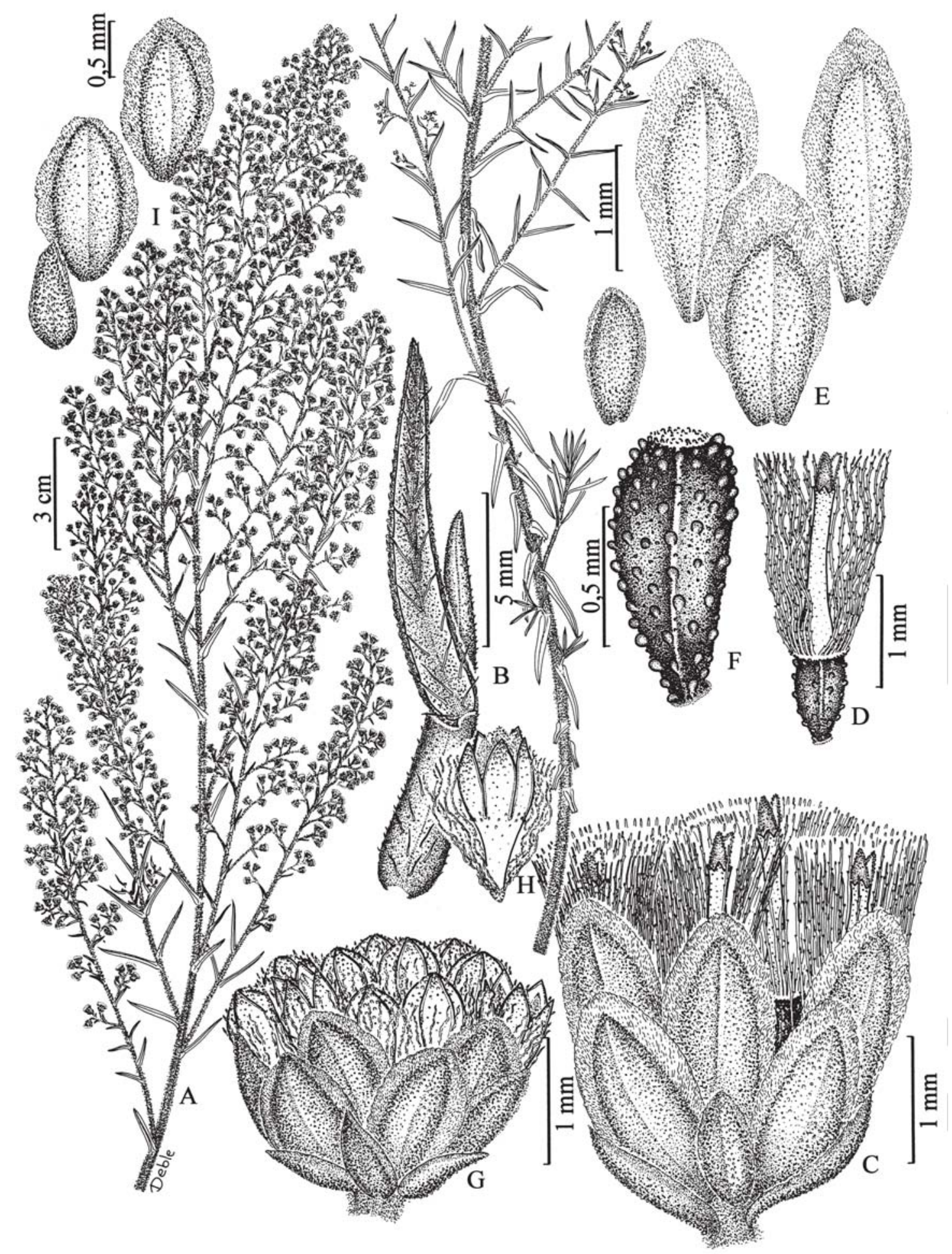

Fig. 3. Baccharis multipaniculata Oliveira-Deble \& Deble. A: female branch. B: leaves, abaxial and adaxial surface. C: female capitulum. D: female flower. E: female involucral bracts. F: achene. G: male capitulum. H: male flower. I: male involucral bracts (A-F, Reitz \& Klein 12222 holotypus HBR; G-I, Rambo s. n. paratypus PACA). 
Achenes yellowish or light brown, obovoidellipsoid 1-1.2 mm long, laterally slightly compressed, with 5 longitudinal ribs and biseriate glandular hairs. Male capitula hemispheric, 2-2.5 $\mathrm{mm}$ long, $2.5 \mathrm{~mm}$ wide; flowers 12-16; involucre 2-2.5 mm long and wide. Involucral bracts in 2-3 series, apex obtuse or slightly acute; outer bracts ovate, 1$1.6 \mathrm{~mm}$ long, 0.8-1 mm wide; inner bracts ovate to broadly elliptic, 1.5-2 mm long, 1-1.2 $\mathrm{mm}$ wide. Male corolla 1-1.2 mm long, apically with 5 lanceolate lobes. Anthers 0.5$0.7 \mathrm{~mm}$ long. Style not or slightly exceeding the corolla, 1-1.5 mm long; style branches short, attached or free. Pappus white, uniseriate, 1-1.5 mm long; bristles 15-20.

Distribution and habitat: Known only from two collections from Rio Grande do Sul and Santa Catarina (Brazil).

Obs.: Baccharis multipaniculata belongs to sect. Tarchonanthoides (Heering) Cuatrec. The new species is related to $B$. coridifolia DC., differing by subcordate leaves (versus narrowed or rounded), by female capitulescence with more capitula (versus female capitulescence with less capitula), by broadly campanulate, $2.5-3 \mathrm{~mm}$ long and wide female capitula (versus broadly cylindrical to narrowly campanulate, 6-10 $\mathrm{mm}$ long, 2.5-3.5 mm wide), by 1.3-1.5 mm long female corolla (versus 2.8-4 mm long) and by the pappus of the female flowers with 35-40 bristles (versus 100-130 bristles).

Additional specimen examined. BRAZIL. Rio Grande do Sul: São Francisco de Paula, Itaimbezinho, in paludosis dumetosis, $\sigma^{x}, 12-$ II1956, B. Rambo s.n. (PACA 58.571).

Etymology: The specific epithet refers to the capitulescence of many terminal panicles.

\section{Baccharis nassauvioides Oliveira-Deble \& Deble, sp. nov.}

Fig. 4

Suffrutex ramosus; caulibus apice dense foliosus, postremo cicatricosis. Folia obovata, obovatospathulata vel orbiculata, 4-9 mm longa, 3-6 mm lata, dense imbricata, trinervata, sessilia, concolora, paucidentata vel integra, apice rotundata, basi obtusa vel attenuata. Capitula sessilia vel breviter pedicellata in spicis foliorum disposita. Involucrum femineum cilyndraceum, 4,5-6,5 mm altum, 0,8-1,5 $\mathrm{mm}$ crassum, 1-2-florum. Bracteis involucri 4-5seriatis, leviter acutis, margine hyalinis; externis ovatis ad ellipticis, 2-2,5 mm longis, 0,8-1 mm latis; mediis oblongis vel lanceolatis, 3-4 mm longis, 0,8-1 $\mathrm{mm}$ latis; intimis anguste oblongis, $4-5 \mathrm{~mm}$ longis, 0,8-1 mm latis. Flores feminei corolla tubularfiliformia, 3-3,2 mm longa, apice obtuse 5-dentata. Stylus 3,8-4,5 mm longus; rami styli linearlanceolati, 0,8-1 mm. Pappus albus, multiseriatus, 2,2-3 mm longus; setis pappi 90-112. Achaenia glabra, castanea, 10-12-costata, oblonga, 2-2,5 mm longa. Capitula mascula anguste campanulata, 4,5$5 \mathrm{~mm}$ alta, 1,5-2 $\mathrm{mm}$ crassa; involucrum mascularum cilyndraceum, 3,5-4 mm altum, 1-1,2 $\mathrm{mm}$ crassum, 5-7-florum. Bracteis involucri 3-4seriatis, leviter acutis; externis ovatis, 1-2 mm longis, 0,6-0,8 mm latis; mediis ovatis ad orbiculatis, 2-2,5 $\mathrm{mm}$ longis, 1,3-1,6 mm latis; intimis lanceolatis, 2,8$3 \mathrm{~mm}$ longis, 1-1,2 mm latis. Flores mascula corolla tubuloso-campanulata, 3,4-3,7 mm longa, apice 5lobata; lobulis lanceolatis, 1-1,2 mm longis. Antherae 1-1,1 mm longae. Stylo 3,5-4,5 mm longo; rami styli connati, longi exserti. Pappus albus, biseriatus, 3-3,5 mm longus; setis pappi 48-56.

Typus: Brazil. Rio de Janeiro: Itatiaia, Prateleiras, $2.200 \mathrm{~m}$ s.m., ㅇ, 8-V-1975, A. M. Camerish 57a (holotypus RB).

Subshrub; branching erect; apex densely leafy. Leaves obovate, obovate-spathulate to nearly circular, 4-9 mm long, 3-6 mm wide, densely imbricate, sessile, concolorous, resinosous, 3-veined from near the base, margin with few short obtuse teeth (rarely entire), apex rounded, base obtuse to narrowed. Capitula sessile or shortly pedunculate, in leaf axils. Female capitula and involucre narrowly cylindrical, 4.5-6.5 mm long, 0.8-1.5 mm wide; flowers 1-2; involucral bracts in 4-5 series, apex slightly acute, margin hyaline; outer bracts ovate to elliptic, 2-2.5 mm long, 0.8-1 mm wide; median bracts oblong to lanceolate, 3-4 mm long, 


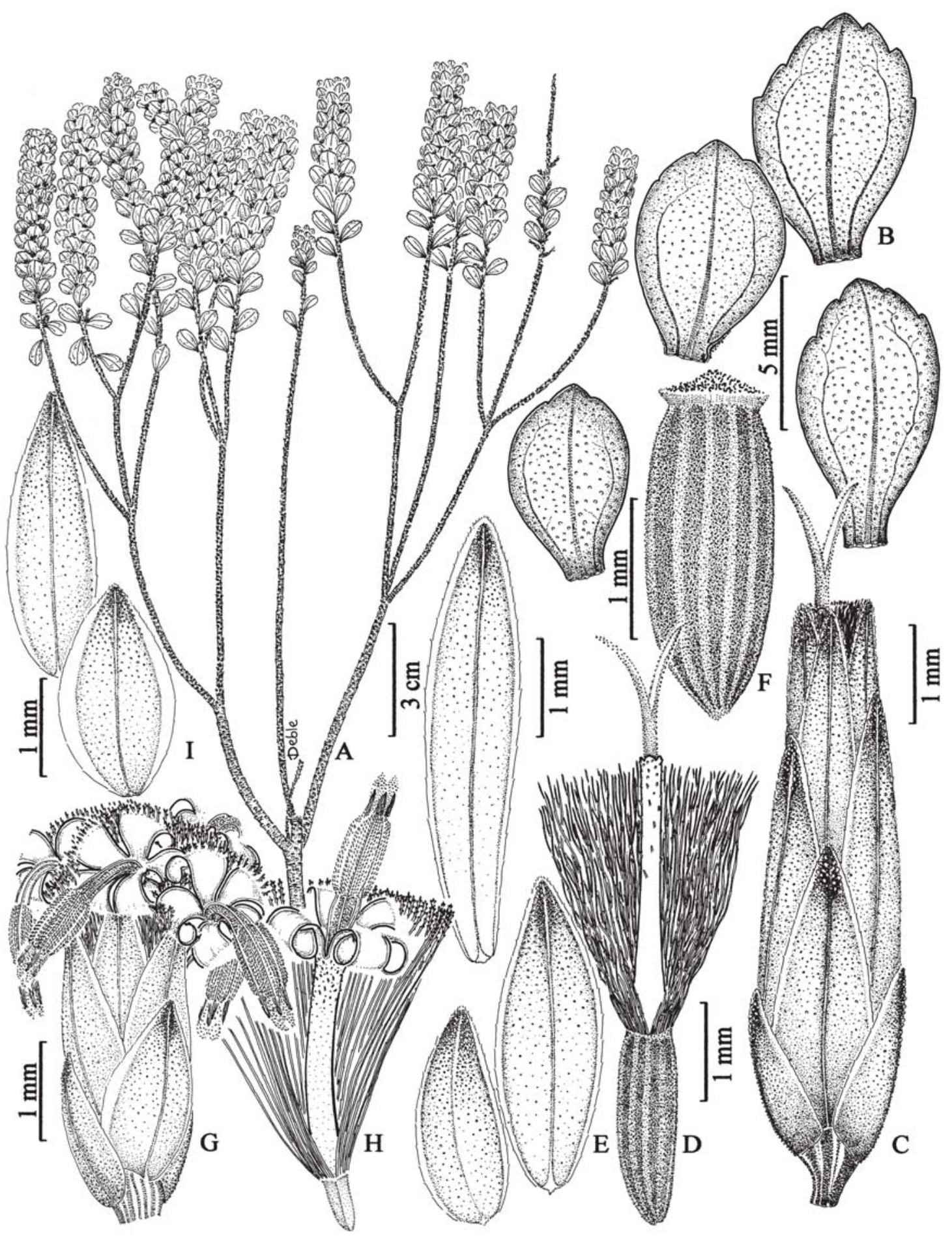

Fig. 4. Baccharis nassauvioides Oliveira-Deble \& Deble. A: female branch. B: leaves. C: female capitulum. D: female flower. E: female involucral bracts. F: achene. G: male capitulum. H: male flower. I: male involucral bracts (A-F, Camerish 57 a holotypus RB; G-I, Camerish 57b paratypus RB). 
0.8-1 mm wide; inner bracts narrowly oblong, 4-5 mm long, 0.8-1 mm wide. Female corolla tubular-filiform, 3-3.2 mm long, apically with 5 short rounded teeth. Style exceeding the corolla, 3.8-4.5 mm long; branches linearlanceolate, 0.8-1 mm long. Pappus white, multiseriate, 2.2-3 mm long; bristles 90-112. Achenes oblong, 2-2.5 mm long, glabrous, brownish, 10-12-ribbed. Male capitula narrowly campanulate, 4.5-5 mm long, 1.5-2 mm wide; flowers 5-7; involucre cylindrical, 3.5-4 mm long, 1-1.2 mm wide; involucral bracts in 3-4 series, apex slightly acute; outer bracts ovate, $1-2 \mathrm{~mm}$ long, 0.6-0.8 mm wide; median bracts ovate to nearly circular, 2-2.5 $\mathrm{mm}$ long, 1.3-1.6 mm wide; inner bracts lanceolate, 2.8-3 mm long, 1-1.2 mm wide. Male corolla 3.4-3.7 mm long, apically with 5 lanceolate lobes, 1-1.2 mm long. Anthers 1$1.1 \mathrm{~mm}$ long. Style exceeding the corolla, 3.5$4.5 \mathrm{~mm}$ long; style branches short, attached. Pappus white, biseriate, 3-3.5 mm long; bristles 48-56.

Distribution and habitat: Baccharis nassauvioides is known only from the type collection in the highest elevations of the Serra de Itatiaia (Itatiaia), Rio de Janeiro (Brazil).

Obs.: Baccharis nassauvioides belongs to Baccharis sect. Cylindricae ser. Axillaris. The new species is close to Baccharis aracatubensis Malag. \& Hatsch. ex G. M. Barroso, and differs by obovate, obovatespathulate to nearly circular, 4-9 mm long and 3-6 mm wide leaves (versus oblong leaves, 10-15 mm long, 4-6 mm wide), by 4.5-6.5 mm long and 0.8-1.5 mm wide female involucres (versus 7-9 mm long, 2.5-2.8 $\mathrm{mm}$ wide), with 1-2 flowers (versus 3-5) and by a 2.2-3 mm long pappus of the female flowers (versus 6-8 $\mathrm{mm}$ long). Baccharis nassauvioides is also similar to $B$. serrula Sch.Bip., differing by imbricate, 4-9 $\mathrm{mm}$ long and 3-6 mm wide leaves (versus not imbricate, $12-15 \mathrm{~mm}$ long, 2-4 mm wide) with a few short obtuse teeth or rarely with entire margin (versus margin with 6-12 acute teeth).

Additional specimen examined. BRAZIL. Rio de Janeiro: Itatiaia, Prateleiras, $2.200 \mathrm{~m}$ s.m., $\sigma^{x}$, 8-V-1975, A. M. Camerish 57b [fragment, in pocket, together with Camerish 57a].

Etymology: The specific epithet refers to the habit similar to many species of the genus Nassauvia Comm. (Asteraceae).

\section{Baccharis suberectifolia Oliveira-Deble \& Deble, sp. nov.}

Fig. 5

Suffrutex 0,8-1,5 m altus; caulibus erectus, inferne simplicibus. Folia linearia, 15-35 mm longa, 1-3 mm lata, alterna (internodiis 4-15 mm longis), subadpressa, 1-3-nervata, sessilia, integra, punctulato-glandulosa, apice acuta, basi trunculata. Capitula pedicellata in panicula racemosis disposita. Capitula feminea oblonga, 7-8 mm alta, 2$3 \mathrm{~mm}$ crassa; involucrum oblongum, 5-5,5 mm altum, 2-3 mm crassum, 5-6-florum. Bracteis involucri 3-seriatis, acutis vel obtusis, margine hyalinis; externis ovato-lanceolatis ad lanceolatis, 1,5-3 mm longis, 1-1,2 mm latis; mediis et intimis oblongis, 4-4,5 mm longis, 1-1,5 mm latis. Flores feminei corolla tubulosa, 3,4-3,6 mm longa, ad apicem glanduloso-dentata. Stylus 4-4,2 mm longus; rami styli lanceolati, 0,6-0,7 mm longi. Pappus fulvus, multiseriatus, 3,5-4,5 mm longus; setis pappi 55-60. Achaenia castanea, leviter compressa, 2-2,5 $\mathrm{mm}$ longa, puberula, cum pilis geminis et pappilis vestita. Capitula mascula campanulata vel hemisphaerica, 3,5-4 mm alta et crassa; involucrum 2,5-3 mm altum, 2,5-3,5 mm crassum, 15-20-florum. Bracteis involucri 2-3-seriatis, acutis vel rotundatis; externis ovatis vel lanceolatis, 1,5-2,5 mm longis, 11,2 mm latis; intimis ovatis vel orbiculatis, 2,2-2,5 mm longis, 1-2 mm latis. Flores mascula corolla tubuloso-campanulata, 2,5-3 mm longa, apice 5lobata; lobulis lanceolatis, 0,6-0,7 mm longis, cum pilis geminis et papillis vestita. Antherae 0,7-0,8 mm longae. Stylus 3-3,3 mm longus; rami styli breves, connati. Pappus albus vel fulvus, uniseriatus, 1,5-2 mm longus; setis pappi 16-22.

Typus: Brazil. Paraná: Palmeira, Rod. BR-277, rio Papagaios, campo limpo, ereta, capítulos alvescentes, ㅇ, 11-II-1988, G. Hatschbach \& S. Ginzbarg 51875 (holotypus MBM). 


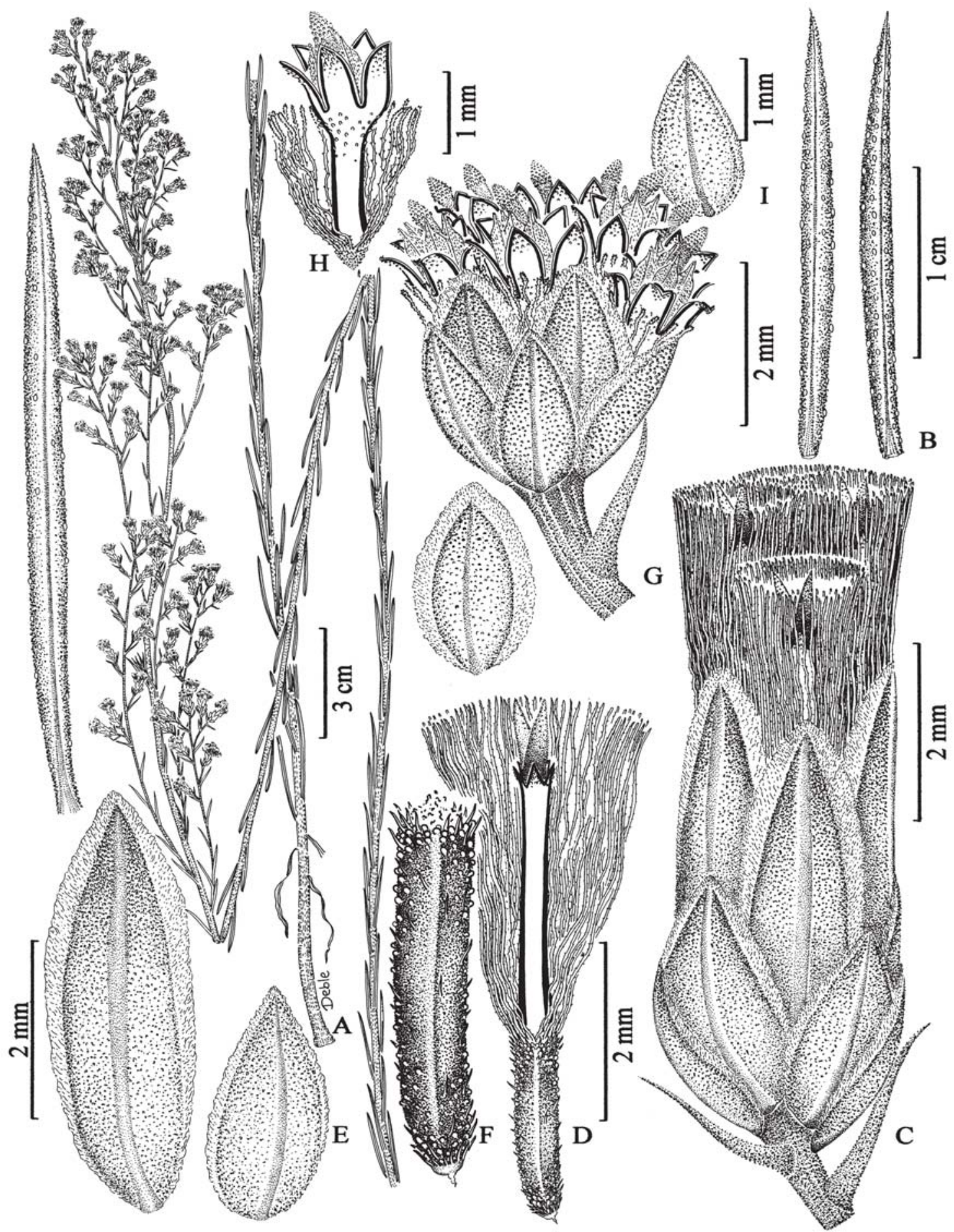

Fig. 5. Baccharis suberectifolia Oliveira-Deble \& Deble. A: female plant. B: leaves. C: female capitulum. D: female flower. E: female involucral bracts. F: achene. G: male capitulum. H: male flower. I: male involucral bracts (A-F, Hatschbach \& Ginzbarg 51875 holotypus MBM; G-I, Hatschbach \& Ginzbarg 51876 paratypus MBM). 
Subshrub; stems single, erect, branching only in the capitulescence, 0.8-1.5 m tall. Leaves appressed to the stem, alternate, concolorous, linear, 15-35 mm long, 1-3 mm wide, appearing 1-3-veined, sessile, glandular, margin entire, apex acute, base truncate, internodes 4-15 mm. Capitula pedunculate, borne in terminal panicles. Female capitula broadly cylindrical, 7-8 mm long, $2-3 \mathrm{~mm}$ wide; flowers 5-6; involucre 5-5.5 mm long, 2-3 mm wide; involucral bracts in 3-series, apex acute or obtuse; outer bracts ovatelanceolate to lanceolate, 1.5-3 mm long, 1-1.2 mm wide; median and inner bracts oblongs, 4$4.5 \mathrm{~mm}$ long, 1-1.5 mm wide. Female corolla tubular, 3.4-3.6 mm long, apically with 5 papillose teeth. Style exceeding the corolla, 4$4.2 \mathrm{~mm}$ long; branches lanceolate, 0.6-0.7 mm long. Pappus yellow, in several series, 3.5-4.5 mm long; bristles 55-60, basally connate, persistent. Achenes dark brown, 5-ribbed, slightly compressed laterally, 2-2.5 mm long, with biseriate glandular hairs and twin hairs. Male capitula campanulate to hemispheric, 3.5-4 mm long and wide; flowers 15-20; involucre $2.5-3 \mathrm{~mm}$ long, $2.5-3.5 \mathrm{~mm}$ wide; involucral bracts in 2-3-series, acute to rounded; outer bracts ovate to lanceolate, 1.5$2.5 \mathrm{~mm}$ long, 1-1.2 mm wide; inner bracts ovate to nearly circular, 2.2-2.5 mm long, 1-2 $\mathrm{mm}$ wide. Male corolla 2.5-3 mm long, apically with 5 lanceolate lobes, 0.6-0.7 mm long. Anthers 0.7-0.8 mm long. Style not or slightly exceeding the corolla, 3-3.3 mm long; style branches short, attached or free. Pappus white or yellow, uniseriate, 1.5-2 mm long; bristles 16-22.

Distribution and habitat: The new species is known only from southern Paraná (Brazil), in grassland near rio Papagaios, Palmeira and Campo Largo.

Obs.: Baccharis suberectifolia belongs to sect. Tarchonanthoides. The new species is related to $B$. coridifolia DC., differing by simple stems branching only in the capitulescence (versus branching stems), by erect leaves that are appressed to the stem (versus erect-patent or patent, not apressed to stem), by acute or obtuse female involucral bracts (versus apex rounded) and by the pappus of female flowers with 55-60 bristles (versus 100-130). Baccharis suberectifolia is also similar to $B$. erigeroides DC., differing by linear, $15-35 \mathrm{~mm}$ long and 1-3 mm wide leaves (versus oblong, 20-50 mm long, 3-8 $\mathrm{mm}$ wide), by $4-15 \mathrm{~mm}$ long internodes (versus $10-40 \mathrm{~mm}$ ), by broadly cylindrical female capitula 7-8 mm long and 2-3 mm wide (versus campanulate, 9-11 mm long, 7-8 mm wide); as well as by number of flowers in female capitula (5-6 versus 15-20).

Additional specimens examined. BRASIL. Paraná: Campo Largo, rio Papagaios, campo limpo, 930 m s.m., ㅇ, 23-II-1960, G. Hatschbach 6759 (MBM). Palmeira, rio Papagaios, campo limpo seco, ereta, capítulos creme, ơ , 11-II-1988, G. Hatschbach \& S. Ginsbarg 51876 (MBM).

Etymology: The specific epithet refers to the frequently erected leaves.

\section{Acknowledgements}

The authors are indebted to the curators of HBR, MBM, PACA and RB, for making specimens available for study. We also thank two anonymous referees for review of the manuscript.

\section{Bibliography}

OLIVEIRA, A. S. de, DEBLE, L. P., SCHNEIDER, A. A. \& J. N. C. MARCHIORI. 2006. Checklist do gênero Baccharis L. para o Brasil (AsteraceaeAstereae). Balduinia 9: 17-27.

OLIVEIRA-DEBLE, A. S. de. 2008. Classificação infragenérica e atualização nomenclatural das espécies brasileiras de Baccharis L. (AsteraceaeAstereae). Tese de Pós-graduação em Engenharia Florestal, Universidade Federal de Santa Maria, Santa Maria, Brasil. 256 pp. 